\title{
CICLO BIOLÓGICO Y CAPACIDAD PREDADORA DE Podisus nigrispinus (DALLAS, 1851) EN LARVAS DE Spodoptera frugiperda (WALKER, 1857), S. eridania (CRAMER, 1782) Y Galleria sp. (LINNAEUS, 1756) EN CONDICIONES DE LABORATORIO

\author{
BIOLOGICAL CYCLE AND PREDATORY CAPACITY OF \\ Podisus nigrispinus (DALLAS, 1851) IN LARVAE OF \\ Spodoptera frugiperda (WALKER, 1857), S. eridania (CRAMER, 1782) AND \\ Galleria sp. (LINNAEUS, 1756) UNDER LABORATORY CONDITIONS
}

\author{
David Alva Romero ${ }^{1}$, Carmen Calderón Arias $^{2}$ y Katheryn Georget Pisfil Colchado ${ }^{3}$
}

\begin{abstract}
Resumen
Podisus nigrispinus (Hemiptera: Pentatomidae) es un depredador e importante controlador biológico. El objetivo de esta investigación fue determinar en condiciones de laboratorio el ciclo biológico y la capacidad predadora de ninfas y adultos de Podisus nigrispinus (predador) utilizando larvas de Spodoptera frugiperda, S. eridania y Galleria sp. (presas). Los experimentos fueron realizados en condiciones controladas de temperatura $\left(23{ }^{\circ} \mathrm{C} \pm 5{ }^{\circ} \mathrm{C}\right)$ y humedad relativa $(72 \% \pm$ $6 \%$ ). Los tratamientos consistieron en un individuo de cada estado de desarrollo de $P$. nigrispinus (ninfa II, III, IV, V y el adulto) suministrados con 30 larvas de cada presa durante todo el ciclo de vida, haciendo un total de veinte tratamientos. Los parámetros evaluados para el ciclo de vida fueron: número de días de vida por estado ninfa y longevidad en estado adulto y ciclo de vida del individuo en relación a cada presa. Para la capacidad predadora los parámetros fueron: número de larvas depredadas y número máximo de presas consumidas por estado ninfa y estado adulto. Los datos obtenidos en las evaluaciones fueron analizados utilizando la prueba de Kruskall-Wallis. Los resultados señalaron que el número de días de vida para ninfa fue de 14.6, 33.35 y 36.95 y para adulto de 29.5, 33.35 y 36.95; siendo el ciclo de vida de 44.10, 66.70 y 73.20 días para $S$. frugiperda, $S$. eridania y Galleria sp., respectivamente. El consumo de larvas en estado ninfa de $P$. nigrispinus fue de 21.90, 20.20 y 13.05; en estado adulto fue de 29.5, 33.35 y 36.95 para $S$. frugiperda, $S$. eridania y Galleria sp. respectivamente. La máxima capacidad predadora de $P$. nigrispinus en estado ninfa fue de 28.00 y en estado adulto fue 50.00, ambas con larvas de $S$. frugiperda. En consecuencia, se demostró que $P$. nigrispinus en estado adulto tuvo una mayor capacidad predadora cuando se le suministró larvas de $S$. nigrispinus.
\end{abstract}

Palabras clave: capacidad predadora, ciclo biológico, Podisus, Galleria, Spodoptera.

\section{Abstract}

Podisus nigrispinus (Hemiptera: Pentatomidae) is a predator and important biological controller. The objective of this research was to determine under laboratory conditions the biological cycle and the predatory capacity of nymphs and adults of Podisus nigrispinus (predator) using larvae of Spodoptera frugiperda, S. eridania and Galleria sp. (dams). The experiments were carried out under controlled conditions of temperature $\left(23{ }^{\circ} \mathrm{C} \pm 5{ }^{\circ} \mathrm{C}\right)$ and relative humidity $(72 \% \pm 6 \%)$. The treatments consisted of an individual from each stage of development of $P$. nigrispinus (nymph II, III, IV, V and the adult) supplied with 30 larvae from each prey throughout the life cycle, making a total of twenty treatments. The parameters evaluated for the life cycle were: number of days of life per nymph state and longevity in adult state and life cycle of the individual in relation to each prey. For the predatory capacity the parameters were: number of predated larvae and maximum number of prey consumed per nymph and adult stage. The data obtained in the evaluations were analyzed using the Kruskall-Wallis test. The results indicated that the number of days of life for nymphs were 14.6, 33.35 and 36.95 and for adults 29.5, 33.35 and 36.95, with a life cycle of 44.10, 66.70 and 73.20 days for $S$. frugiperda, S. eridania and Galleria sp. respectively. The consumption of larvae in nymph stage of P. nigrispinus was 21.90, 20.20 and 13.05; and in adult stage it was 29.5, 33.35 36.95 for $S$. frugiperda, $S$. eridania and Galleria sp. respectively. The maximum predatory capacity of $P$. nigrispinus in nymph stage was 28.00 and in adult stage it was 50.00 , both with larvae of 
S. frugiperda. Consequently, it was shown that $P$. nigrispinus in adult stage had a greater predatory capacity when $S$. nigrispinus larvae were fed to it.

Key words: predatory activity, biological cycle, Podisus nigrispinus, Galleria, Spodoptera.

\section{Introducción}

El control biológico de plagas, parte del Manejo Integrado de Plagas (MIP), es una alternativa que busca la mejor combinación de métodos de control para una plaga de insectos. Los insecticidas químicos son un componente del MIP que deben usarse de manera menos perjudicial para el control biológico (Reddy, 2017). Con los métodos de introducción de entomófagos, con métodos inoculativos, o el incremento de población natural de predadores, parasitoides y patógenos, se pueden reducir las poblaciones de plagas y mantener el equilibrio ambiental sin la utilización, o con un menor uso, de insecticidas (Symondson et al., 2002; Matos et al., 2004).

Una de las plagas insectiles más importantes en el cultivo de hortalizas es Spodoptera sp. (Lepidoptera: Noctuidae), una polilla polífaga, siendo los adultos nocturnos y no visibles durante el día (Meagher et al., 2008). El principal daño es causado por larvas durante sus últimos instares; ellas raspan, esqueletizan o destruyen totalmente el follaje y, en algunos casos, afectan también el fruto (Mamani, 2012). Ante ello, el uso de especies predadoras para controlar estas plagas es una de las mejores opciones de solución (Matos et al., 2004).

En el control biológico, el insecto predador Podisus nigrispinus (Dallas, 1851) (Hemiptera: Pentatomidae) es un importante agente de control ya que consume diferentes plagas de lepidópteros (Torres et al., 2006) y otras especies (Denez et al., 2014). Ellos se alimentan de una gran variedad de plagas de insectos (Schaeffer \& Panizzi, 2000). Por esta razón, estos enemigos naturales han sido criados con diferente dietas (presas) como Tenebrio molitor, (Coleoptera: Tenebrionidae), Musca domestica (Diptera: Muscidae), Bombyx mori (Lepidoptera: Bombycidae), Agrotis ypsilon, Felia experta (Beserra et al., 1995; Zanuncio et al., 1996b; Molina-Rugama et al., 1997; Nascimento et al., 1997; Jusselino et al., 2001), además a las dietas artificiales (Zanuncio et al., 1996a; De Clercq et al., 1998; Coracini et al., 1999; Rojas et al., 2000; Saavedra et al., 2009).

El ciclo de los pentatómidos depende de la cantidad de alimento ingerido (Serrão et al., 2006). Estos predadores pueden reducir su taza de reproducción para mantener sus límites de supervivencia (Ramalho et al., 2008). El peso ganado, calidad y cantidad del alimento puede afectar el número de huevos (Zanuncio et al., 2002).

El género Podisus es criado en laboratorios con diferentes tipos de presa, tales como Galleria sp., Spodoptera sp., y dietas artificiales, en las que, en cada una, se observan cambios en el período de duración de su ciclo. Al utilizar larvas de Tenebrio monitor y Musca domestica para la crianza de P. distinctus se evidenciaron diferencias en la ganancia de peso del pentatómido (Matos et al., 2004), por lo que el alimento sería un parámetro a tomar en cuenta en programas de crianza intensiva.

El estudio del ciclo biológico de Podisus nigrispinus a base de diferentes tipos de larvas permite implementar una crianza controlada, ya que la dieta puede influir en el tiempo de desarrollo, tanto de la ninfa como del adulto. Sin embargo, estos chinches también pueden comportarse en su hábitat natural como fitófagos, alimentándose de plantas para compensar la escasez de presas (Mathias et al., 2009). Para la capacidad predadora de Podisus se tiene que tener en cuenta que el primer instar no se alimenta, a excepción de agua, comportándose como filtrador por unos días (Saavedra et al., 1996). Comportamiento semejante presentan las especies de Podisus maculiventris (Nájera et al., 2016) y Podisus modestus. El canibalismo se observa solo en casos de falta de alimento, al igual que en Podisus sculptus. Las ninfas de Podisus se agrupan solo en caso de que la larvapresa sea de un tamaño considerable; comportamiento similar se ha registrado en Podisus connexivus alimentadas con Bombix mori y Tenebrio molitor (Nascimento et al., 1996). El estudio de la capacidad predadora de Podisus nigrispinus permite también conocer el mínimo suficiente de larvas necesarias para el desarrollo de ninfas con el fin de disminuir costos en la producción y evitar sobregastos.

Los heteroptera en su hábitat natural son principalmente chupadores de savia, pero algunas especies de la familia Pentatomidae desarrollaron hábitos depredadores y son comunes durante los brotes de insectos (Ferreira et al., 2008). Liberaciones de depredadores en sistemas agrícolas y forestales representan una etapa crítica en los programas de control biológico porque las condiciones adversas, incluida la escasez de presas puede reducir su potencial biológico y reproductivo (Fialho et al., 2009). Por tanto, en el presente trabajo se trató de determinar el ciclo biológico y la capacidad predadora de Podisus nigrispinus sobre Spodoptera frugiperda, Spodoptera eridania y Galleria sp., en condiciones de laboratorio.

\section{Materiales y Métodos}

La investigación se realizó en las instalaciones del Laboratorio de Control Biológico del Instituto de Biotecnología de la Universidad Nacional Pedro Ruiz Gallo, Lambayeque, a una temperatura de $23{ }^{\circ} \mathrm{C} \pm 5^{\circ} \mathrm{C}$, 
con $72 \% \pm 6 \%$ de humedad relativa, medidas con termómetro ambiental e higrómetro respectivamente.

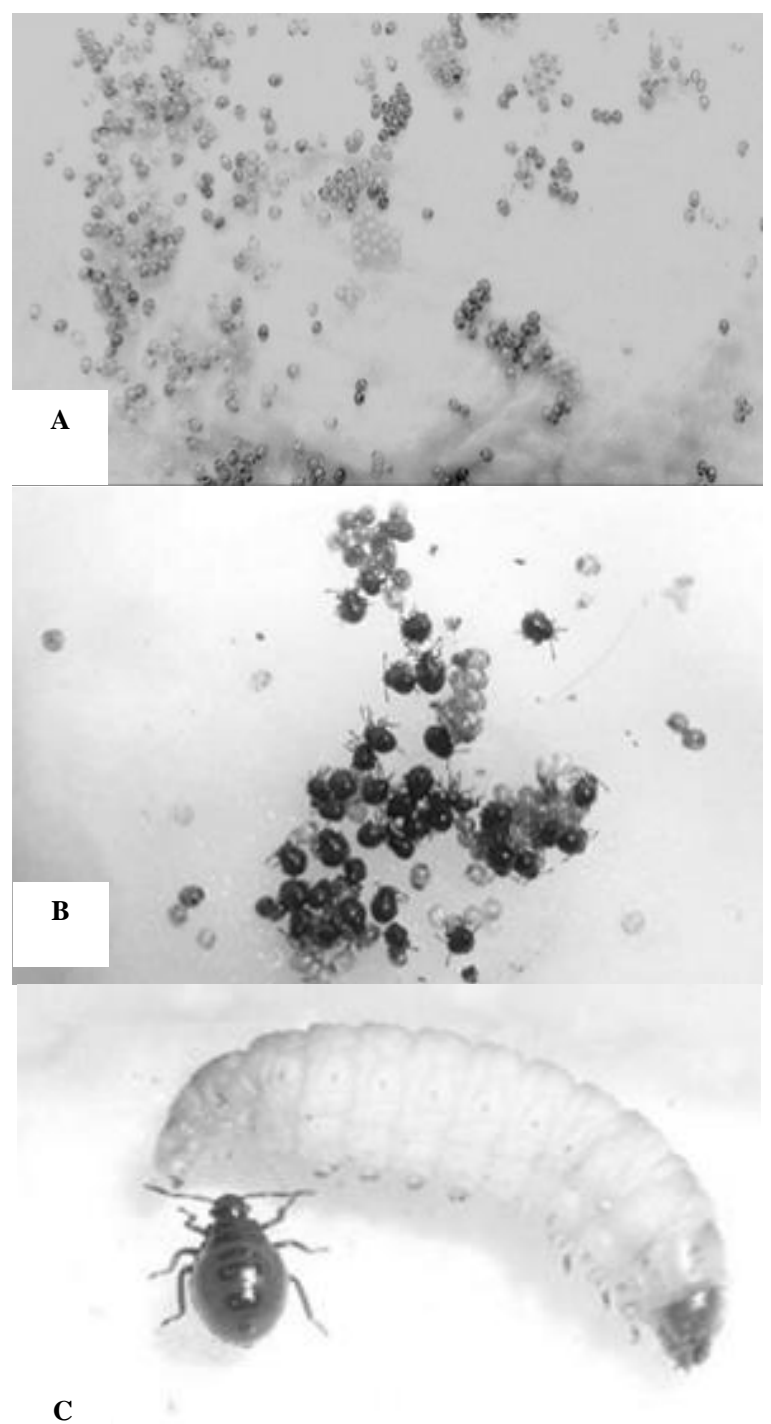

Figura 1. Ciclo de Podisus nigrispinus. A: Huevos. B: Ninfa I. C: Ninfa II. Fotografías: Pisfil, 2017.

Para el estudio de la biología se dispusieron de 30 huevos tomados de la misma postura de Podisus nigrispinus, con una edad de pocos minutos posteriores a la postura; luego fueron separados individualmente en un taper pequeño cerrado con tela gaza; cada contenedor con un algodón húmedo, con una o dos larvas de Galleria sp. Cada día se registró tamaño, color, duración de vida por estado ninfa y estado adulto. Para el tamaño, se separaron los datos obtenidos de 15 individuos hembras y 15 machos.

Para la evaluación de la capacidad predadora del estado ninfal se utilizaron táperes herméticos de $8 \mathrm{~cm}$ de diámetro que fueron rotulados para la identificación correspondiente; en la base de cada taper se colocó algodón humedecido con agua, una ninfa de $P$. nigrispinus de II instar, luego se agregaron larvas como alimento (presas) de tres especies: Spodoptera frugiperda, Spodoptera eridania y Galleria sp., cuyas larvas fueron proporcionadas por el laboratorio. Se realizó el estudio de 30 individuos por cada especie de presa de un tamaño y peso similar. Se examinó diariamente, a una misma hora, el número de larvas que consumían o se encontraban muertas, se procuró dejar diariamente en los táperes 4 larvas vivas del mismo tamaño para que las ninfas de $P$. nigrispinus tengan alimento a disposición y poder evaluar su capacidad predadora. El registro de estos datos se efectuó en el transcurso de la duración de los cinco estadios ninfales de $P$. nigrispinus.

Para medir la capacidad predadora de $P$. nigrispinus se realizó un estudio a partir del segundo estadio ninfal. Las presas se consideraron muertas en cuanto perdieron movilidad, se tornan a un color oscuro, y su cuerpo pierde tonicidad. $P$. nigrispinus al poseer un aparato picador-chupador, actúa, introduciendo su estilete, absorbiendo el contenido interno de la presa, inmovilizándola y matándola a los pocos minutos.

Se realizó la separación individual a partir de ninfas de segundo instar, se consideró como inicio esta fase por la pérdida del comportamiento gregario y el desarrollo locomotor que les permiten moverse con rapidez en busca de alimento.

\section{Resultados}

Ciclo biológico

\section{HUEVO}

El huevo es esférico, de 0.5 - 1.00 mm de diámetro, de color plateado brillante al momento de la postura oscureciéndose conforme se acerca la etapa de eclosión, de un color oscuro en el día 4, anaranjadorojizo en el día 5, adoptando forma ovalada (Figura 1A). La hembra produce una postura de 10 a 50 huevos. La incubación necesita ligera humedad.

\section{NINFA}

Ninfa I: Las ninfas de primer instar tienen una longitud total entre $1.10-1.20 \mathrm{~mm}$, de cabeza negra, tórax oscuro, abdomen posterior de color rojizo con manchas negras (Figura 1B). Poseen poco movimiento, y se comportan como filtradores, alimentándose básicamente de agua y residuos de huevos y azucares disueltos en el agua, semejante a como actúan en campo (Mathias et al., 2009). La Primera muda de exoesqueleto, de $P$. nigrispinus, ocurre entre los $6 \pm 1$ días. La muda dura entre 15 - 90 minutos por individuo.

Ninfa II: Tiene un tamaño entre $1.85-2.85 \mathrm{~mm}$, su desarrollo locomotor es mayor permitiéndole encontrar a su presa, a partir de esta fase se considera predador (Figura 1C).

Ninfa III: Posee un tamaño entre 3.30 - $3.70 \mathrm{~mm}$, es de color negro con manchas naranjas brillantes (Figura 2A).

Ninfa IV: Puede presentar colores de naranja, o rojo combinado con negro. Pueden alcanzar un tamaño entre 
$5.00-6.40 \mathrm{~mm}$, reconocibles por tener un aspecto plano ensanchado (Figura 2B).

Ninfa V: Tiene un tamaño entre $6.70-8.30 \mathrm{~mm}$ de longitud; destaca por sus colores brillantes y llamativos, con manchas rojas, anaranjadas o blancas (Figuras 2C y 3A).

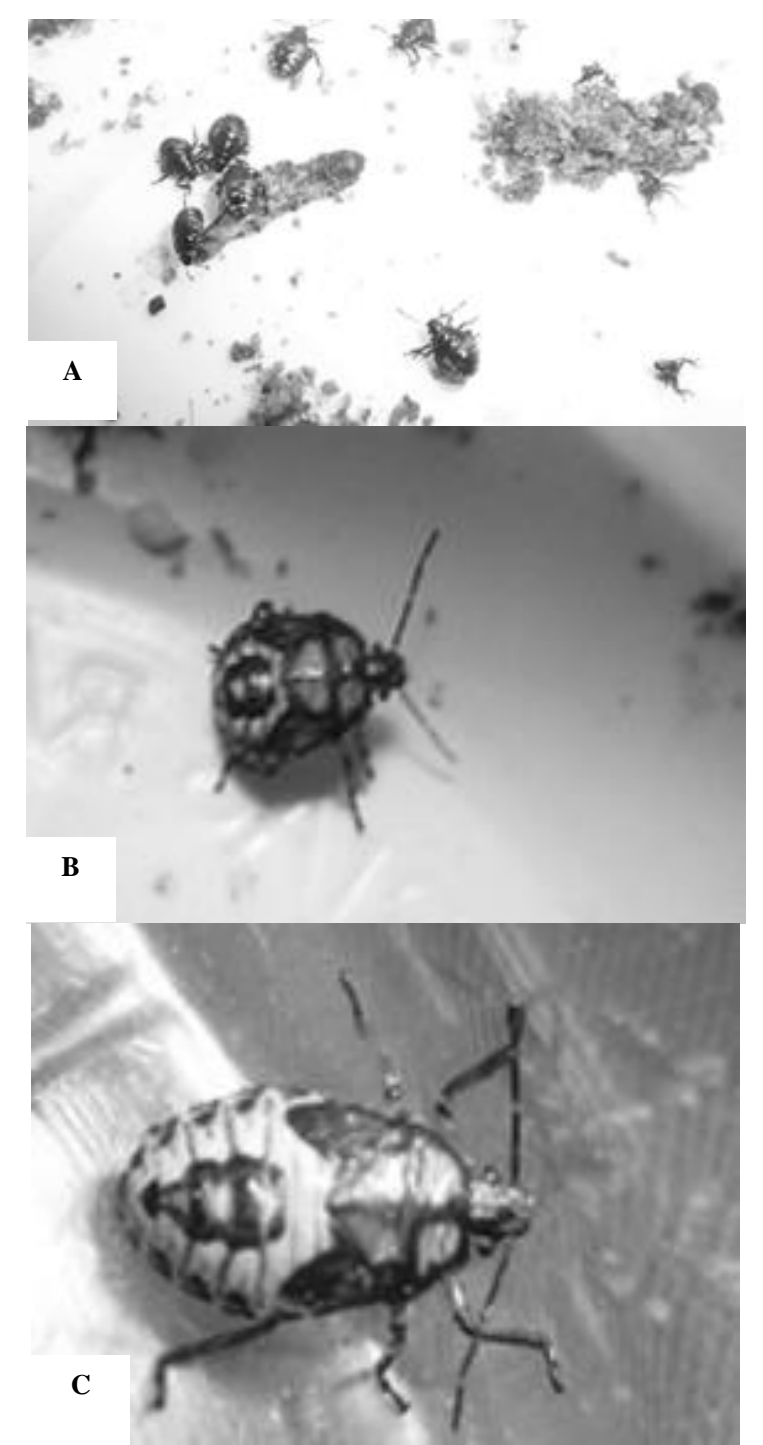

Figura 2. Estadíos ninfales de Podisus nigrispinus. A: Ninfa III. B: Ninfa IV. C: Ninfa V. Fotografías: Alva.2017.

\section{Adulto}

Presentan dimorfismo sexual (Figura 3B), los machos son de menor tamaño que las hembras, en promedio los machos tienen $9.40 \mathrm{~mm}$ de longitud y $11.15 \mathrm{~mm}$ las hembras. La duración de la cópula oscila entre 12 - 72 horas. El macho tiende a demorar unos días más que la hembra en alcanzar la madurez sexual.

LONGEVIDAD

Se evaluó la duración de los estados ninfa y adulto, en días, de $P$. nigrispinus, en 10 individuos alimentados con larvas de Spodoptera frugiperda, Spodoptera eridania y Galleria sp.

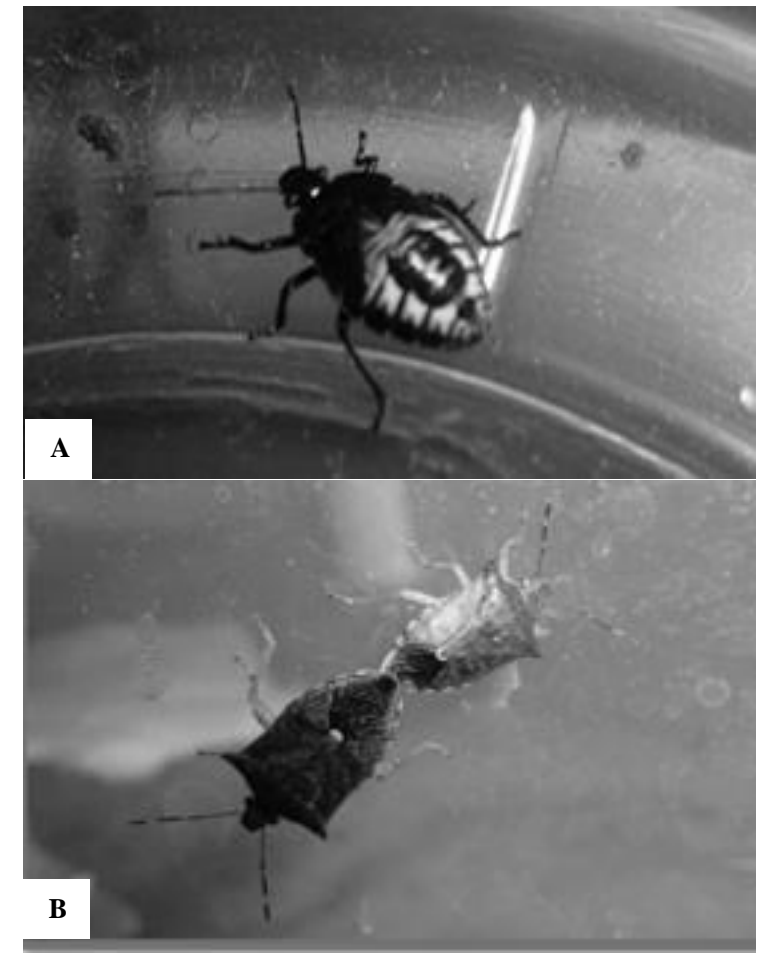

Figura 3. Estadío y copulación de Podisus nigrispinus. A: Ninfa V. B: Cópula de adultos. Fotografías: Alva, 2017.

A una temperatura de $23{ }^{\circ} \mathrm{C} \pm 5{ }^{\circ} \mathrm{C}$, con $72 \% \pm 6 \%$ de humedad relativa, alimentados con larvas de Galleria sp., para los individuos de P. nigrispinus se registroó una media de 5.7 días para la eclosión de los huevos y para las ninfas se registraron: 3.3, 3.1, 3.0, 4.6 y 5.7 días, respectivamente, para cada instar del primero al quinto. En los adultos, se obtuvo un promedio de 24.4 días de longevidad (Figura 4).

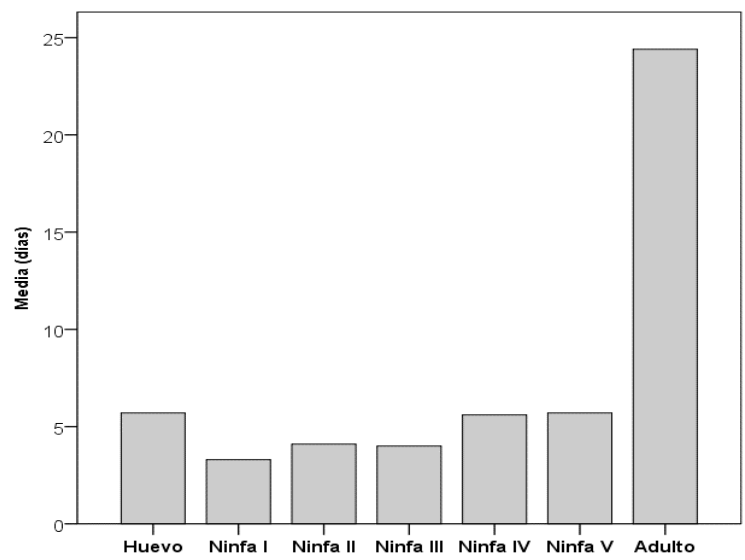

Figura 4. Longevidad de Podisus nigrispinus alimentados con larvas de Galleria sp.

En los tratamientos con 30 individuos de P. nigrispinus, teniendo como presas larvas de Spodoptera frugiperda, S. eridania y Galleria sp., se registraron medias de duración en estado ninfal de $14.60,33.35$ y 36.95 días respectivamente; en los 
adultos fue de 29.50, 33.35 y 36.95 días respectivamente; la mayor longevidad total se observó en el grupo alimentado con Galleria sp., alcanzando una media de 73.90 días, un periodo más corto con $S$. eridania de 66.70 días; en $S$. frugiperda el ciclo es más corto, con 44.10 días (Tabla 1).

Tabla 1. Estado ninfal y longevidad de adultos de Podisus nigrispinus en días, alimentados con larvas de Spodoptera frugiperda, Spodoptera eridania y Galleria sp., a una temperatura de $23{ }^{\circ} \mathrm{C} \pm 5{ }^{\circ} \mathrm{C}$, con $72 \% \pm 6 \%$ de humedad relativa.

\begin{tabular}{|c|c|c|c|c|}
\hline & & \multicolumn{3}{|c|}{ Presa } \\
\hline & & 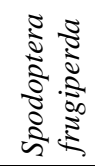 & 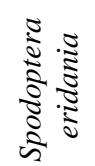 & 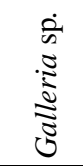 \\
\hline \multirow{4}{*}{$\begin{array}{l}\text { 或 } \\
\text { 哥 }\end{array}$} & Media & 14.60 & 33.35 & 36.95 \\
\hline & Mínimo & 14.00 & 17.00 & 15.00 \\
\hline & Máximo & 15.00 & 45.00 & 50.00 \\
\hline & $\begin{array}{l}\text { Desviación } \\
\text { estándar }\end{array}$ & 0.50 & 8.66 & 10.77 \\
\hline \multirow{4}{*}{ 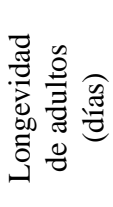 } & Media & 29.50 & 33.35 & 36.95 \\
\hline & Mínimo & 10.00 & 17.00 & 15.00 \\
\hline & Máximo & 41.00 & 45.00 & 50.00 \\
\hline & $\begin{array}{l}\text { Desviación } \\
\text { estándar }\end{array}$ & 10.29 & 8.66 & 10.77 \\
\hline 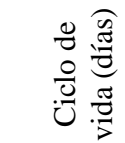 & Media & 44.10 & 66.70 & 73.90 \\
\hline
\end{tabular}

A través de prueba de Kruskall-Wallis ( $\mathrm{p}=0.05)$, se determinaron diferencias significativas para el estadío ninfal y longevidad de adultos de $P$. nigrispinus. Para las ninfas que tuvieron como presa a $S$. frugiperda se determinó un periodo significativo más corto de duración de su estado ninfal comparado con el resto de grupos, no existiendo diferencias entre los grupos de S. eridania y Galleria sp. (Figura 5). El grupo de S. frugiperda presentó una baja dispersión de datos, siendo la desviación estándar de 0.50 .

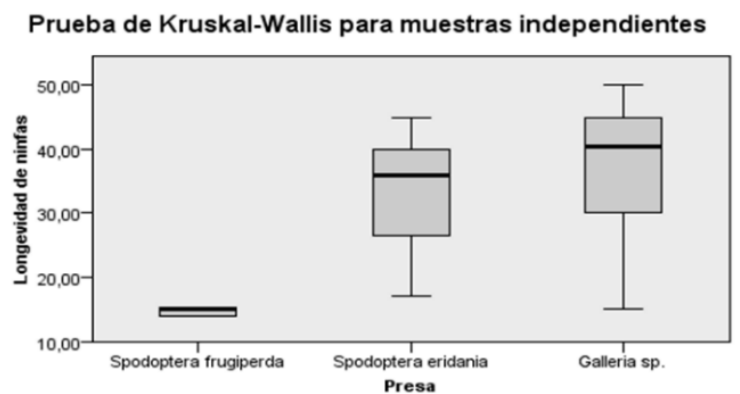

Figura 5. Longevidad de ninfas de Podisus nigrispinus, alimentadas con larvas de Spodoptera frugiperda, S. eridania y Galleria sp.
La longevidad de adultos de P. nigrispinus alimentados con Galleria sp., alcanzó un mayor periodo de vida significativo (36.95 días en promedio) comparada la de los adultos alimentados con Spodoptera frugiperda, que alcanzó 29.50 días de vida (Figura 6); con S. eridania como presa se obtuvo un valor intermedio entre los tres grupos de 33.35 días como promedio.

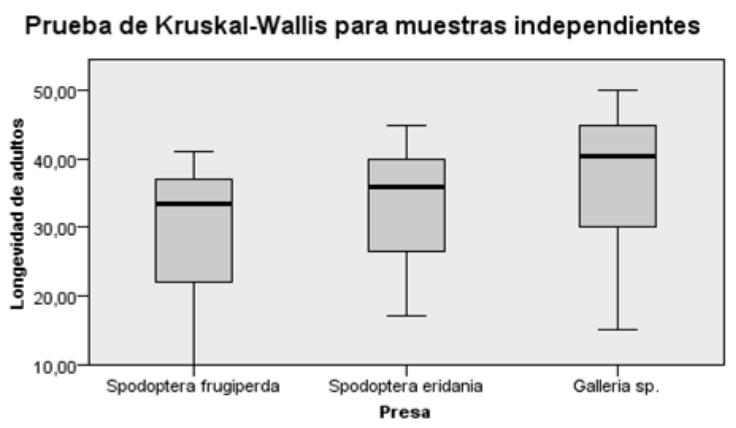

Figura 6. Longevidad de adultos de Podisus nigrispinus alimentados con larvas de Spodoptera frugiperda, S. eridania y Galleria sp.

Capacidad predadora

El número de larvas predadas depende de la especie y el estadio en que se encuentre el predador. Se obtuvo la capacidad predadora del estado ninfal de $P$. nigrispinus en Spodoptera frugiperda, Spodoptera eridania y Galleria sp. una media de $21.90,20.20$ y 13.05 larvas consumidas respectivamente, mientras que el estado adulto de $P$. nigrispinus consumió un total de $32.8,18.1$ y 14.55 larvas (Tabla 2).

Tabla 2. Larvas de Spodoptera frugiperda, S. eridania y Galleria sp. predadas por ninfas y adultos de Podisus nigrispinus.

\begin{tabular}{|c|c|c|c|c|}
\hline & & \multicolumn{3}{|c|}{ Presa } \\
\hline & & 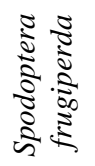 & 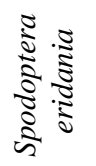 & 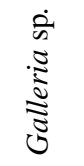 \\
\hline \multirow{5}{*}{ 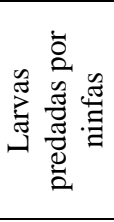 } & Media & 21.90 & 20.20 & 13.05 \\
\hline & Mínimo & 17.00 & 18.00 & 11.00 \\
\hline & Máximo & 28.00 & 22.00 & 16.00 \\
\hline & $\begin{array}{l}\text { Desviación } \\
\text { estándar }\end{array}$ & 3.48 & 1.28 & 1.76 \\
\hline & Varianza & 12 & 2 & 3 \\
\hline \multirow{5}{*}{ 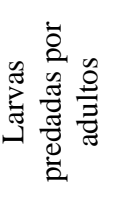 } & Media & 32.80 & 18.10 & 14.55 \\
\hline & Mínimo & 18.00 & 9.00 & 7.00 \\
\hline & Máximo & 50.00 & 25.00 & 22.00 \\
\hline & $\begin{array}{l}\text { Desviación } \\
\text { estándar }\end{array}$ & 8.98 & 4.66 & 4.33 \\
\hline & Varianza & 81 & 22 & 19 \\
\hline
\end{tabular}

Para el caso de larvas predadas por ninfas se utilizó la prueba de Kruskal-Wallis $(\mathrm{p}=0.05)$, donde 
S. frugiperda fue la presa y $P$. nigrispinus tuvo mayor actividad predadora (21.90 larvas consumidas), caso contrario, Galleria sp. fue la presa donde se registró menor actividad predadora (13.05 larvas consumidas) (Figura 7).

\section{Prueba de Kruskal-Wallis para muestras independientes}

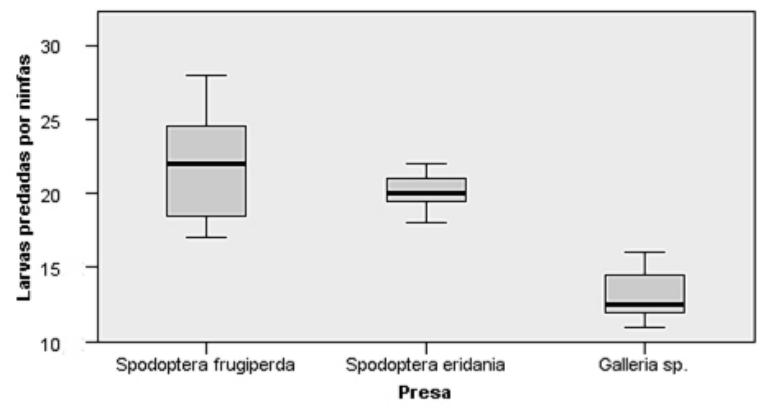

Figura 7. Larvas predadas de Spodoptera frugiperda, S. eridania y Galleria sp. por ninfas de P. nigrispinus.

Los adultos de $P$. nigrispinus predaron un mayor número de larvas $S$. frugiperda $(\mathrm{X}=32.80)$, en relación a las larvas de $S$. eridania (18.10) y Galleria $\mathrm{sp.}(\mathrm{X}=$ 14.55) (Figura 8).

Las ninfas de P.nigrispinus tuvieron mayor capacidad predadora por días que los adultos, en las larvas de los dos grupos de Spodoptera spp. En cambio, el comportamiento de ninfas y adultos de $P$. nigrispinus fue ligeramente mayor y casi similar entre ninfas y adultos alimentándose con larvas Galleria sp. (Figura 9). Las ninfas predaron una media de $1.50,0.61$ y 0.35 larvas por día de S.frugiperda, S. eridania y Galleria sp., los adultos predaron 1.11, 0.54 y 0.39 larvas por día.

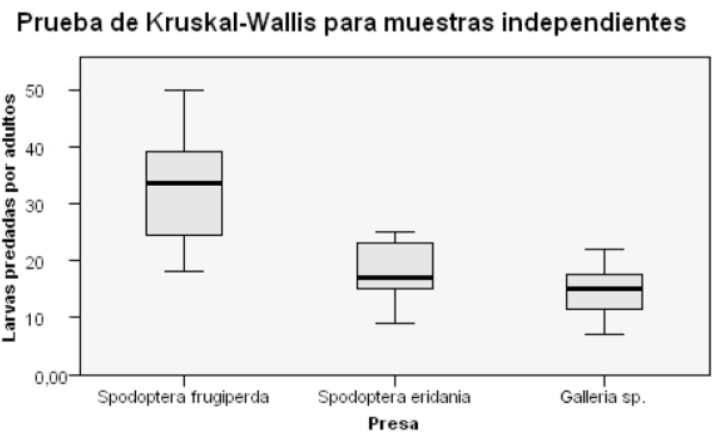

Figura 8. Larvas predadas de Spodoptera frugiperda, S. eridania y Galleria sp. por adultos de P. nigrispinus.

\section{Discusión}

La menor longevidad obtenida en adultos de P. nigrispinus, con Spodoptera frugiperda como presa, es explicado con el trabajo de Oliveira (Oliveira et al., 2004) que registra variaciones en las hembras, con una menor longevidad con respecto a los machos, por lo que, el uso de esta larva puede ser utilizada para alcanzar la madurez sexual del individuo de forma rápida; luego, cambiar la presa a Galleria sp. con el fin de prolongar la longevidad del adulto para obtener mayor cantidad de posturas.

La capacidad predadora de P. nigrispinus fue mayor en adultos de Spodoptera frugiperda, S. eridania y Galleria sp.; este resultado coincide con el trabajo de Pereira et al. (2005) quienes detallan que el consumo de la presa se debe al factor de saciación de $P$. nigrispinus con determinadas larvas.

La capacidad predadora por día es mayor en las ninfas que en los adultos de Podisus nigrispinus para Spodoptera frugiperda y S. eridania, por lo cual sería conveniente la liberación de ninfas de $P$. nigrispinus en programas de control biológico de estas plagas.

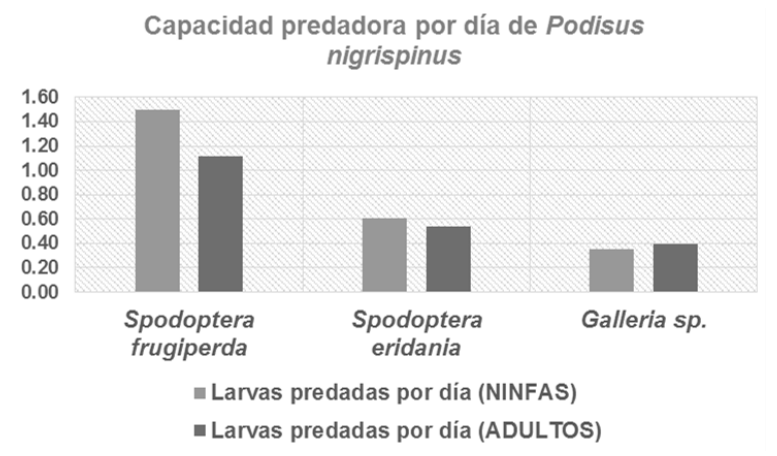

Figura 9. Capacidad predadora por día de ninfas y adultos de Podisus nigrispinus sobre Spodoptera frugiperda, S. eridania y Galleria sp.

\section{Conclusiones}

Podisus nigrispinus tiene cinco estadios ninfales, el primer instar se alimenta de agua, a partir del segundo instar se considera predador. El tamaño alcanzado por adultos de $P$. nigrispinus está relacionado directamente al sexo; las hembras miden entre 10.20 - $12.15 \mathrm{~mm}$ mientras que los machos 8.15 - $10.30 \mathrm{~mm}$ de longitud total.

A un rango de temperatura de $23{ }^{\circ} \mathrm{C} \pm 5{ }^{\circ} \mathrm{C}$ y a una humedad relativa de $72 \% \pm 6 \%, P$. nigrispinus presenta un ciclo biológico de 44.10, 66.70 y 73.90 días teniendo como presa a Spodoptera frugiperda, S. eridania y Galleria sp., respectivamente. La duración del estadio ninfal es de 14.6, 19.4 y 24.8 días alimentado con larvas S. frugiperda, S. eridania y Galleria sp., respectivamente; la longevidad de adultos presenta 29.5, 33.35, y 36.95 días en promedio, respectivamente.

La mayor capacidad predadora fue observada en ninfas, superando a los adultos. Se registraron 21.90, 20.2, y 13.05 larvas consumidas por ninfas de $P$. nigrispinus sobre $S$.frugiperda, S. eridania y Galleria sp., respectivamente; mientras, en los adultos se registraron 32.8, 18.1, y 14.55 larvas consumidas. Las ninfas son más voraces que los adultos teniendo como presa a $S$. frugiperda y $S$. eridania. 


\section{Literatura citada}

Beserra E.B., Zanuncio T.V., Zanuncio J.C. \& Santos G.P. 1995. Desenvolvimento de Supputius cincticeps (Heteroptera, Pentatomidae) alimentado com larvas de Zophobas confusa, Tenebrio molitor (Coleoptera: Tenebrionidae) e Musca domestica (Diptera: Muscidae). Rev. Brasil. Zool., 12(3): 725-733. https://doi.org/10.1590/S0101-81751995000300026.

Coracini M.D., Vilela E.F., Junior P.M., Zanuncio J.C. \& Furtado M.F. 1999. Localização e aceitação de dieta artificial contendo feromônio sexual pelo predador Podisus nigrispinus (Dallas) (Heteroptera: Pentatomidae). An. Soc. Entomol. Bras., 28(4): 687-693. https://doi.org/10.1590/S0301-80591999000400011.

De Clercq P., Merlevede F. \& Tirry L. 1998. Unnatural prey and artificial diets for rearing Podisus maculiventris (Heteroptera: Pentatomidae). Biol. Cont., 12(2): 137-142. https://doi.org/10.1006/bcon.1998.0611.

Denez M.D., Bueno A.F., Pasini A., Bortolotto O.C. \& Stecca C.S. 2014. Biological parameters of Podisus nigrispinus (Hemiptera: Pentatomidae) fed with different soybean insect pests. Annals of Entomological Society of America, Annapolis, 107(5): 967-974. https://doi.org/10.1603/AN14054.

Ferreira J.A.M., Zanuncio J.C., Torres J.B. \& MolinaRugama A.J. 2008. Predatory behaviour of Podisus nigrispinus (Heteroptera: Pentatomidae) on different densities of Anticarsia gemmatalis (Lepidoptera: Noctuidae) larvae. Biocontrol Sci. Techn., 18(7):

711-719. https://doi.org/10.1080/09583150802271220.

Fialho M.D.C.Q., Zanuncio J.C., Neves C.A., Ramalho F.S. \& Serrão J.E. 2009. Ultrastructure of the digestive cells in the midgut of the predator Brontocoris tabidus (Heteroptera: Pentatomidae) after different feeding periods on prey and plants. Ann. Entomol. Soc. Am., 102(1): 119-127. https://doi.org/10.1603/008.102.0113.

Jusselino F.P., Zanuncio J.C., Guedes N.C. \& Fragoso D.B. 2001. Desarrollo y reproducción del predador Brontocoris tabidus (Heteroptera: Pentatomidae) alimentado con larvas de Tenebrio molitor (Coleoptera: Tenebrionidae). Rev. Colomb. Entomol., 27: 45-48.

Mamani G. 2012. Entomología Agrícola. Guía de Prácticas. Universidad Nacional de San Agustín. Arequipa, Perú.

Mathias A., Almeida G., Matiello M.A., Zanuncio-Junior J.S., Vinha T. \& Cola J.C. 2009. Survival and reproduction of Podisus nigrispinus (Hemiptera: Pentatomidae): Effects of prey scarcity and plant feeding. Chilean Journal of Agricultural Research, 69(3): 468472. 58392009000300021

Matos N.F., Nonato O.H., Zanuncio J.C., Mathias H.A., Oliveira I. \& Queiroz F.M. 2004. Ganancia de peso del depredador Podisus distinctus (Heteroptera: Pentatomidae) en combinaciones de las presas Tenebrio molitor (Coleoptera: Tenebrionidae) y Musca domestica (Diptera: Muscidae). Revista de Biología $\quad$ Tropical, 52(1): 101-108. https://www.scielo.sa.cr/scielo.php?script=sci_arttext\&p id=S0034-77442004000100014.

https://revistas.ucr.ac.cr/index.php/rbt/article/view/1475 7.
Meagher R.L., Brambila J. \& Hung E. 2008. Monitoring for exotic Spodoptera species (Lepidoptera: Noctuidae) in Florida. Florida Entomologist, 91(4): 517-522. https://doi.org/10.1653/0015-4040-91.4.517.

Molina-Rugama A.J., Zanuncio J.C., Torres J.B. \& Zanuncio T.V. 1997. Longevidad y fecundidad de Podisus nigrispinus (Heteroptera: Pentatomidae) alimentado com Musca domestica (Diptera: Muscidae) y frijol. Rev. Biol. Trop., 45(3): 1125-1130. https://revistas.ucr.ac.cr/index.php/rbt/article/view/2113 5.

Nájera M.D., Ordóñez G.M., Ríos V.C., Berlanga-Reyes D.I., Acosta-Muñiz C.H., Zamudio-Flores P.B. \& JacoboCuéllar J.L. 2016. Depredación por Podisus maculiventris (Say) sobre larvas de Choristoneura rosaceana (Harris). Acta zoológica mexicana, 32(2):

147-152. https://doi.org/10.21829/azm.2016.322941.

Nascimento E.C., Zanuncio J.C., Menin E.F. \& Paulo S.F. 1996. Biological, morphological and behavioral aspects of Podisus sculptus distant adults (Heteroptera, Pentatomidae). Rev. Bras. Zool., 13(1): 151-157. https://doi.org/10.1590/S0101-81751996000100015.

Nascimento E.C., Zanuncio J.C., Picanço M.C. \& Zanuncio T.V. 1997. Desenvolvimento de Podisus sculptus Distant, 1889 (Heteroptera: Pentatomidae) em Bombyx mori (Lepidoptera: Bombycidae) e Tenebrio molitor (Coleoptera: Tenebrionidae). Rev. Brasil. Biol., 57: 195201.

Oliveira H.N., Pratissoli D., Pedruzzi E.P. \& Espindula M.C. 2004. Desenvolvimento do predador Podisus nigrispinus alimentado com Spodoptera frugiperda e Tenebrio molitor. Pesq. agropec. bras., 39(10): 947-951. http://dx.doi.org/10.1590/S0100-204X2004001000001.

Pereira I.A., Ramalho F.S. \& Zanuncio J.C. 2005. Susceptibility of Podisus nigrispinus (Dallas) (Heteroptera: Pentatomidae) to gammacyhalothrin under laboratory condictions. Scientia Agricola, Piracicaba, 62(5): 478-482. https://doi.org/10.1590/S010390162005000500012

Ramalho F.S., Mezzomo J., Lemos W.P., Bandeira C.M., Malaquia J.B., Silva J.P.S., Leite G.L.D. \& Zanuncio J.C. 2008. Reproductive strategy of Podisus nigrispinus females under different feeding intervals. Phytoparasitica, 36(1): 30-37. https://doi.org/10.1007/BF02980745.

Reddy G.V. 2017. Integrated Management of Insect Pests on Canola and other Brassica Oilseed Crops. CABI, Wallingford, Oxfordshire, UK.

Rojas M.G., Morales J.A. \& King E.G. 2000. Two meridict diets for Perillus bioculatus (Heteroptera: Pentatomidae), a predador of Leptinotarsa decemlineata (Coleoptera: Chrysomelidae). Biol. Cont., 17(1): 92-99. https://doi.org/10.1006/bcon.1999.0780.

Saavedra J.L., Zanuncio J.C., Zanuncio T.V. \& Guedes R.N.C. 2009. Prey capture ability of Podisus nigrispinus (Dallas) (Heteroptera: Pentatomidae) reared for successive generations on meridic diets. Journ. Appl. Entom., $\quad 121(1-5)$ : $327-330$. https://doi.org/10.1111/j.1439-0418.1997.tb01414.x. 
Saavedra J.L., Zanuncio J.C., Zanuncio T.V. \& Santos. 1996. Desarrollo ninfal de Podisus nigrispinus (Heteroptera: Pentatomidae) en alimentación mixta de dieta artificial y larvas de Musca domestica (Diptera: Muscidae). Brenesia, $\quad 1996(45-46)$ : 177-182. http://biblioteca.museocostarica.go.cr/articulo.aspx?id=2 $517 \&$ art $=3607401$.

Schaeffer C.W. \& Panizzi A.R. 2000. Heteroptera of economic importance. CRC Press, New York.

Serrão J.E., Zanuncio J.C., Bauce E., Lemos W.P. \& Ramalho F.S. 2006. Diet affects reproduction and number of oocytes per ovary of the predator Podisus nigrispinus (Dallas) (Heteroptera: Pentatomidae). Anim. Biol., 56: 279-287. https://doi.org/10.1163/157075606778441840.

Symondson W.O., Sunderland K.D. \& Greenstone M.H. 2002. Can generalist predators be effective biocontrol agents? Ann. Rev. Entomol., 47: 561-594. https://doi.org/10.1146/annurev.ento.47.091201.145240.

Torres J.B., Zanuncio J.C. \& Moura M.A. 2006. The predatory stinkbug Podisus nigrispinus: biology, ecology and augmentative releases for lepidopteran larval control in Eucalyptus in Brazil. Biocontrol News and Information, 27(15): 1-15. http://dx.doi.org/10.1079/PAVSNNR20061015.
Zanuncio J.C., Saavedra J.L., Zanuncio T.V. \& Santos G.P. 1996a. Desarollo y reproducción de Supputius cincticeps (Heteroptera: Pentatomidae) en dieta artificial por dos generaciones. Revista de Biología Tropical, 44: 247-251. URI: http://hdl.handle.net/10669/25759.

Zanuncio J.C., Saavedra J.L., Zanuncio T.V. \& Santos G.P. 1996b. Incremento en el peso d ninfas y adultos de Podisus nigrispinus (Heteroptera: Pentatomidae) alimentados com dos tipos de larvas. Revista de Biología Tropical, $44(3) / 45(1)$ : 241-245. https://revistas.ucr.ac.cr/index.php/rbt/article/view/2201 0.

Zanuncio J.C., Molina A.J., Santos G.P. \& Ramalho F.S. 2002. Effect of body weight on fecundity and longevity of the stinkbug predator Podisus rostralis. Pesq. Agropec. Bras., $37(9)$ : 1225-1230. http://dx.doi.org/10.1590/S0100-204X2002000900004.

\footnotetext{
${ }^{1}$ Escuela de Postgrado, Universidad Nacional Toribio Rodríguez de Mendoza. Calle Higos Urco N ${ }^{\circ}$ 342-350-356 - Calle Universitaria $\mathrm{N}^{\circ}$ 3. Chachapoyas, Amazonas (Perú). Autor de correspondencia: alva.romero.david@gmail.com.

${ }^{2}$ Laboratorio de Ciencias Ambientales. Facultad de Ciencias Biológicas, Universidad Nacional Pedro Ruiz Gallo. Calle Juan XXIII S/N, Lambayeque, Lambayeque (Perú). carmencal_2@hotmail.com.

${ }^{3}$ Facultad de Ciencias Biológicas, Universidad Nacional Pedro Ruiz Gallo. Calle Juan XXIII S/N, Lambayeque, Lambayeque (Perú). georgeth_31@ @otmail.com.
} 